\title{
LACTANCIA MATERNA EXCLUSIVA Y FACTORES ASOCIADOS EN MADRES QUE ASISTEN A ESTABLECIMIENTOS DE SALUD DE LIMA CENTRO
}

\author{
EXCLUSIVE BREASTFEEDING AND ASSOCIATED FACTORS IN MOTHERS WHO ATTEND HEALTH \\ ESTABLISHMENTS OF LIMA, PERU \\ Carmen Villarreal-Verde', Maritza Dorila Placencia-Medina², Violeta Alicia Nolberto-Sifuentes ${ }^{3}$
}

\begin{abstract}
RESUMEN
Introducción: La lactancia materna es un proceso biológico natural que es de suma importancia durante el desarrollo de los neonatos. Objetivo: Encontrar la asociación entre la lactancia materna exclusiva y diversos factores en espacios de tiempo diferentes: durante la gestación, durante el parto y en el puerperio en madres que acuden a centros de salud de la DIRIS Lima Centro. Métodos: Se utilizó una encuesta para conocer los factores asociados a la lactancia materna exclusiva como el número de controles prenatales, asistencia a controles pre natales, y si había dado de lactar a su hijo en los últimos 6 meses. Los datos obtenidos fueron procesados estadísticamente obteniendo los valores de Odss Ratio y las variables de asociación. Resultados: De las 140 madres encuestadas con menos de 12 meses de lactancia, 77,85\% manifestó haber acudido a seis controles prenatales, esta actitud tuvo relación estadísticamente significativa con la Lactancia Materna Exclusiva ( $p: 0,012$ ). Se observo que el parto vaginal tiene asociación significativa con la Lactancia Materna. Sin embargo, el uso de fórmulas lácteas podría presentarse como factor de riesgo $(p<0,001)$. Conclusión: Se encontraron tres factores asociados a la LME: asistencia a seis controles pre natales, parto vaginal y como factor de riesgo, el uso de fórmulas lácteas, uno en cada espacio de tiempo, antes, durante y después del parto.
\end{abstract}

Palabras clave: Lactancia materna; Recién nacido; Relaciones madre-hijo; Leche humana (fuente: DeCS BIREME).

\begin{abstract}
Introduction: Breastfeeding is a natural biological process that is of utmost importance during the development of newborns. Objective: To find the association between exclusive breastfeeding and various factors at different times: during gestation, during birth and in the puerperium in mothers who come to DIRIS Lima Centro health centers. Methods: A survey was used to find out the factors associated with exclusive breastfeeding, such as the number of prenatal check-ups, talks given, and whether she had breastfed her child in the last 6 months. The data obtained were statistically processed to obtain the values of Odss Ratio and association variables. Results: Of the 140 mothers surveyed with less than 12 months of lactation, $77.85 \%$ stated that they had attended six prenatal controls, this attitude had a statistically significant relation with Exclusive Breastfeeding ( $p: 0.012$ ). Vaginal delivery was found to be significantly associated with Breastfeeding. However, the use of milk formula could be a risk factor $(p<0.001)$. Conclusion: Three factors were found to be associated with SCl: attendance to six pre-natal controls, vaginal delivery and as a risk factor, the use of milk formula, one in each time period, before, during and after delivery.
\end{abstract}

Key words: Breast feeding; Newborn; Mother-child relations; Human milk (source: MeSH NLM).

\footnotetext{
${ }^{1}$ Facultad de Medicina, Universidad Nacional Mayor de San Marcos, Lima-Perú.

${ }^{2}$ Facultad de Medicina, Universidad Nacional Mayor de San Marcos, Lima-Perú.

${ }^{3}$ Facultad de Ciencias Matemáticas, Universidad Nacional Mayor de San Marcos, Lima-Perú

Citar como: Carmen Villarreal-Verde, Maritza Dorila Placencia-Medina, Violeta Alicia Nolbert-Sifuentes. Lactancia materna exclusiva y factores asociados en madres que asisten a establecimientos de salud de Lima Centro. Rev. Fac. Med. Hum. Abril 2020; 20(2):287-294. DOI 10.25176/RFMH. v20i2.2765

Artículo publicado por la Revista de la Facultad de Medicina Humana de la Universidad Ricardo Palma. Es un artículo de acceso abierto, distribuído bajo los términos de la Licencia Creative Commons: Creative Commons Attribution 4.0 International, CC BY 4.0 (https://creativecommons.org/licenses/by/4.0/), que permite el uso no comercial, distribución y reproducción en cualquier medio, siempre que la obra original sea debidamente citada. Para uso comercial, por favor póngase en contacto con revista.medicina@urp.pe
} 


\section{INTRODUCCIÓN}

La lactancia materna (LM) es un proceso biológico natural en la mujer durante la gestación y su propósito es alimentar y proteger al bebé recién nacido hasta los seis meses de edad. La evidencia de los beneficios de la lactancia materna exclusiva (LME) se han difundido ampliamente, algunos de ellos son su asociación con la disminución de la mortalidad infantil, también la prevención de enfermedades diarreicas agudas (EDA), las infecciones respiratorias agudas (IRA), mejora en el nivel de inteligencia del niño; beneficios para la madre: cáncer de mama y de ovario y prevención de la diabetes $\mathrm{M2}^{(1)}$.

En varias publicaciones se reporta que al alta del hospital donde nace el niño, un alto porcentaje de madres inicia la lactancia el primer díay, sin embargo, en los meses siguientes va decreciendo paulatinamente hasta llegar a niveles muy bajos ${ }^{(2,3)}$, Entre las causas de abandono de la LME, se han encontrado la vuelta de la madre al trabajo o al estudio ${ }^{(4)}$ y la hipogalactia (percepción de producir poca leche y que el niño se quede con hambre) $)^{(4)}$ también la falta de apoyo del padre del bebé o de la familia (ayuda en las tareas domésticas que agotan su energía, produciendo cansancio) $^{(5)}$.

En nuestro país, según la Encuesta Demográfica y de Salud Familiar 2018 del Instituto Nacional de Estadística e Informática ${ }^{(6)}$ la prevalencia de IRA a partir del 2009 hasta el 2018 ha oscilado entre 13,3 y 16,8\%, correspondiendo a 14,4\% para el año 2018, a nivel nacional. En el caso de la prevalencia de EDA, del año 2007 al 2018 ha permanecido relativamente estable, oscilando entre $14,9 \%$ y $10,9 \%$, correspondiendo $10,7 \%$ para el año 2018, a nivel nacional. Con diferencias según áreas, urbana 10,3\% y rural 12,1\%. Sin embargo, según la Oficina de Epidemiología de la Dirección de Redes Integradas de Salud (DIRIS) Lima Centro, de los 14 distritos que la integran, los que tienen mayor porcentaje de casos de IRA, exceptuando neumonía, son los distritos de San Juan de Lurigancho $(41,5 \%)$, Lima Cercado $(14,9 \%)$ y La Victoria $(9,2 \%)$, los demás distritos están por debajo del 4\%.Para los casos de EDA también son mayores los porcentajes de casos para los distritos de San Juan de Lurigancho (34,3\%), Lima Cercado $(20,1 \%)$ y La Victoria $(5,4 \%)$, los otros distritos están por debajo de $3 \%{ }^{(7)}$. Estas morbilidades, como ya se mencionó, están relacionadas a la LME.

Asimismo, se sabe que el parto vía vaginal favorece la $L M E^{(9)}$ sin embargo, el porcentaje de partos por cesárea se incrementó de $26,4 \%$ en el 2008 a $41,0 \%$ para el 2018 en el área urbana (ENDES 2018). La OMS establece que un máximo de intervenciones para partos por cesárea debería ser de $15 \%{ }^{(8)}$.

Se ha reportado también que existe una sólida asociación inversa entre la LM y el cáncer de mama ${ }^{(10)}$, según el Análisis de Situación de Salud del Cáncer del 2018, el cáncer de mama es la principal neoplasia en mujeres en nuestro país ${ }^{(11)}$, esta situación podría reducirse si la LM se promueve y practica con mayor frecuencia y duración, pues son conocidos los efectos de la LM en la prevención del cáncer de mama ${ }^{(1)}$.

Se han publicado numerosos artículos relacionados con la práctica de la lactancia y los factores que puedan favorecerla u obstaculizarla. Un estudio en España informa que la falta de apoyo y de información influye negativamente en la $L M E$, menciona también que algunos profesionales de la salud no están muy informados de los beneficios de la LM, en cambio, las mujeres que han sido preparadas durante la etapa prenatal acerca de los beneficios de la LM, tienen éxito en proporcionar su leche al bebé durante un tiempo mas prolongado y exclusivo(12).

Por las razones expuestas se decidió indagar acerca de los factores que estuvieran asociados a la práctica de la LME en madres que acudían a establecimientos de salud de la DIRIS Lima Centro, en tres distritos en los cuales la prevalencia de IRA y de EDA se encontraban elevadas. El objetivo de este estudio fue encontrar la asociación entre la lactancia materna exclusiva y diversos factores en espacios de tiempo diferentes: durante la gestación, durante el parto y en el puerperio en madres que acuden a centros de salud de la DIRIS Lima Centro.

\section{MÉTODOS}

\section{Diseño}

Se realizó un estudio observacional de corte transversal analítico.

\section{Población y muestra}

Se seleccionaron establecimientos de salud de nivel 1-3, (llamados Centros de Salud) ubicados en tres distritos que integran la DIRIS Lima Centro, debido a que en ellos la prevalencia de IRA y EDA para el 2018 era muy elevada en relación a los otros once distritos de la mencionada DIRIS. En estos distritos predominan familias que corresponden a los estratos $C, D$ y $E^{(13)}$ .Para el cálculo de la muestra se consideró la población de madres que acudía regularmente por mes a los tres centros de salud seleccionados, se calculó un tamaño muestral, obteniéndose una muestra de 129, a la que se añadió $10 \%$ previendo una tasa de rechazo similar, 
al final se logró analizar un total de 140 encuestas.

\section{Procedimientos, variables e instrumentos}

El instrumento utilizado para la obtención de información fue un cuestionario elaborado para conocer los factores que pudieran estar asociados a la práctica de la lactancia materna exclusiva, en tres espacios de tiempo: Antes del parto, es decir, durante la gestación; durante el parto, y después del parto. Las preguntas estaban orientadas acerca del número de controles prenatales a los cuales asistió la madre y las instrucciones recibidas acerca de la lactancia, como su importancia para ella y para el niño, las técnicas de estimulación y de extracción de leche en caso no poder darle directamente, el riesgo que significa utilizar otras leches ofrecidas generalmente en biberones y otros aspectos, en el segundo acápite figuraban preguntas sobre el tipo de parto, contacto precoz del bebé con su madre, alojamiento conjunto y lactancia en el primer día de nacido el bebé. La tercera parte consistía en lo que ocurrió después de parto, como visitas domiciliarias de algún personal de salud, si percibió que tenia poca leche, si tuvo apoyo familiar para poder lactar. Al final se le preguntaba si había dado de lactar a su último hijo hasta los seis meses sin otro alimento adicional. El cuestionario fue validado por jueces expertos y prueba piloto. Se capacitó a un grupo de seis encuestadoras, estudiantes de nutrición para realizar las entrevistas a las madres en cada centro de salud.

Al abordar a una mujer con su hijo esperando atención se le preguntaba si aceptaba responder a las preguntas acerca de la alimentación de su bebé y si este tenía entre 6 y 12 meses, si aceptaba se le pedía firmar el documento de Consentimiento Informado antes de proceder a realizar las preguntas del cuestionario. Una vez concluidas las encuestas en número que correspondía a la muestra total se procedió a la revisión de las mismas para detectar errores u olvido de algún dato, si no se podía corregir o completar, se desechaba la encuesta y se reemplazaba por otras que se volvían a realizar.

\section{Análisis estadístico}

El siguiente paso fue trasladar la información de las encuestas a una base de datos en Excel que luego se transfirió al programa SPSSV22 para su respectivo análisis. Se calculó el Odds Ratio y el nivel de significancia de las asociaciones de variables.

\section{Cuestiones éticas}

El proyecto de investigación fue aprobado por el Comité de Ética de la Facultad de Medicina de la Universidad Nacional Mayor de San Marcos.

\section{RESULTADOS}

\section{Características generales}

Se analizó la información proporcionada por 140 madres de niños menores de 12 meses, el $97 \%$ procedía de tres distritos: Lima Cercado, La Victoria y San Juan de Lurigancho. (ver Tabla 1) El 46,4\% del total de las encuestadas manifestó haberle dado lactancia materna exclusiva (LME) a su hijo.

Entre los establecimientos de salud donde nació el bebé (el último hijo en caso de ser multípara) figura el instituto Materno Perinatal (Maternidad) 27,14\%; en diversos Hospitales de Lima, tanto del MINSA como del Seguro Social, 56.4\%. Hospitales de provincia 5\%, Clínicas particulares 11,4\%. (Tabla 1).

La edad de las madres encuestadas se encontraba en el rango de 20 a 35 años (81,4\%). En el grupo que manifestó haber alcanzado instrucción secundaria (58,6\%), se encontró mayor porcentaje que le proporciona LME a su bebe, en cambio entre las que tienen nivel superior (36,4\%) es mayor el porcentaje que no les da LME a su hijo.

Casi tres cuartas partes (74,3\%) cuenta con pareja, en ellas es ligeramente mayor el porcentaje que les dan LME a sus hijos. De las que no tiene pareja el $69 \%$ no le da la LME.

El $81,4 \%$ de los niños (últimos hijos de las mujeres encuestadas) nacieron con peso normal (2 500g a $4000 \mathrm{~g}$ ), solo un $5,7 \%$ se registró como de bajo peso (menos de $2500 \mathrm{~g}$ ) y un $12,9 \%$ como macrosómico.

\section{Factores antes del parto}

El 77,85\% de las madres manifestó haber acudido a seis controles, este resultado reflejó una relación estadísticamente significativa con la practica de la LME. (p: 0,012) (ver Tabla 2). El análisis de los aspectos tratados durante estos controles con la LME no mostraron significancia estadística. Tampoco se encontró significancia con otros factores como paridad, embarazo planificado y experiencia exitosa de haber dado LME a hijos anteriores (Tabla 2).

\section{Factores durante el parto}

En este grupo se observó que el tipo de parto vaginal tiene asociación significativa con la LME. No se encontró significancia para alojamiento conjunto y contacto piel a piel (ver Tabla 3).

\section{Factores después del parto}

En el periodo del puerperio, no se encontró asociación significativa en los factores que se preguntó a la madre, a excepción del uso de fórmulas lácteas, que podrían interpretarse como factor de riesgo $(p<0,001)$ (Tabla 4). 
Tabla 1. Características de la muestra de las madres encuestadas y establecimientos donde nació su último hijo. Lima 2019.

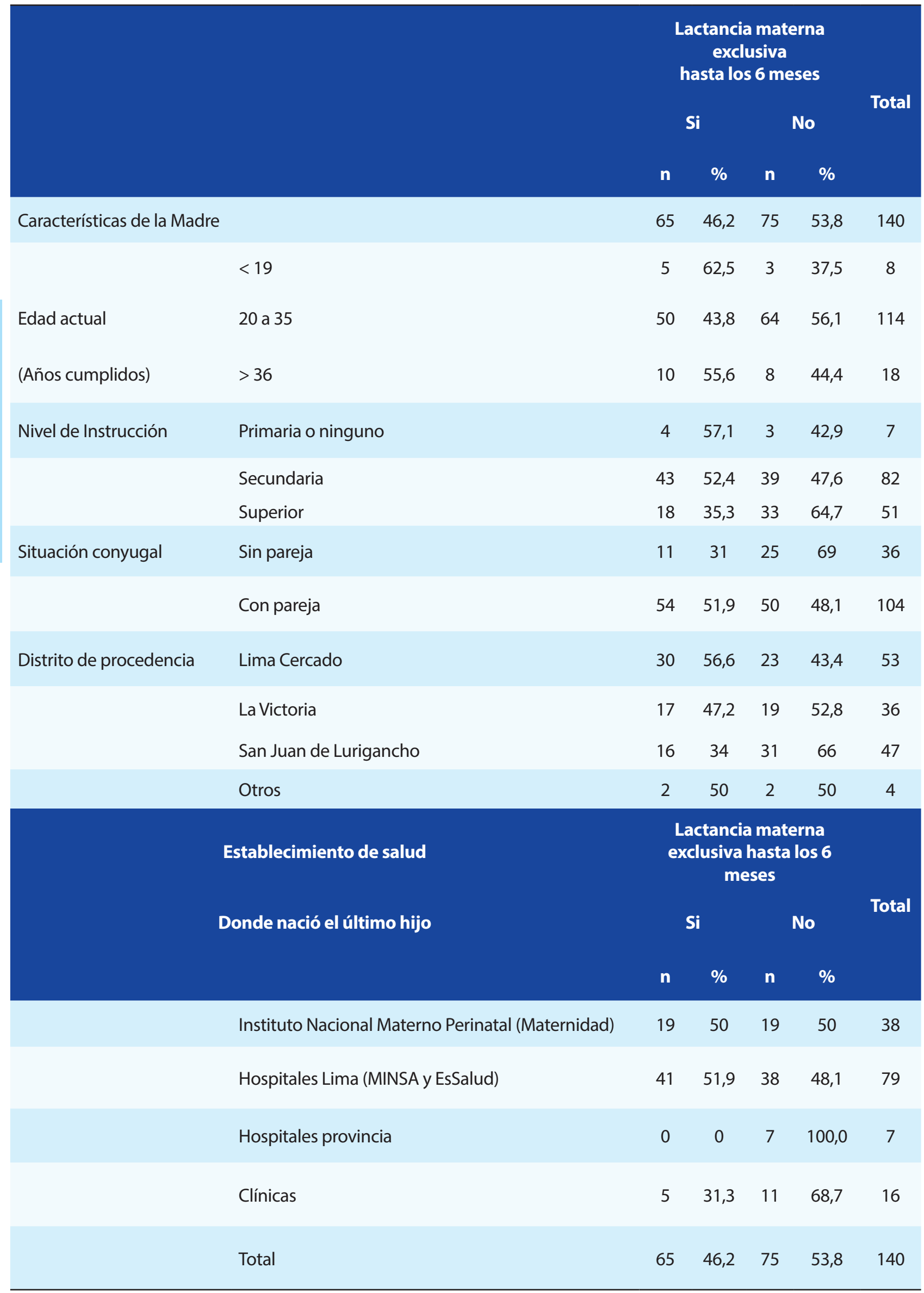


Tabla 2. Nivel de asociación de factores durante la gestación en establecimientos de salud, Lima Centro, junio 2019.

\begin{tabular}{|c|c|c|c|c|c|c|}
\hline Factor & Condición & $\mathbf{n}$ & LME(\%) & OR & IC $95 \%$ & $\underset{\text { significancia }}{\mathbf{p}}$ \\
\hline Asistencia a controles pre natales & $\begin{array}{l}\geq 6 \text { controles } \\
<6 \text { controles }\end{array}$ & $\begin{array}{l}109 \\
31\end{array}$ & $\begin{array}{l}61,5 \\
35,5\end{array}$ & 2,9 & $(1,26-6,65)$ & 0,012 \\
\hline \multicolumn{7}{|c|}{ Aspectos tratados en las sesiones de control prenatal } \\
\hline $\begin{array}{l}\text { Importancia del contacto piel a piel } \\
\text { madre-hijo }\end{array}$ & $\begin{array}{l}\mathrm{SI} \\
\mathrm{NO}\end{array}$ & 120 & $\begin{array}{l}55,8 \\
55,0\end{array}$ & 1,03 & $(0,39-2,67)$ & 0,9446 \\
\hline $\begin{array}{l}\text { Técnicas de estimulación para que el } \\
\text { niño no tenga dificultades al succionar }\end{array}$ & $\begin{array}{l}\text { SI } \\
\text { NO }\end{array}$ & $\begin{array}{l}101 \\
39\end{array}$ & $\begin{array}{l}57,4 \\
51,3\end{array}$ & 1,28 & $(0,61-2,69)$ & 0,5122 \\
\hline Técnicas de amamantamiento & $\begin{array}{l}\text { SI } \\
\text { NO }\end{array}$ & $\begin{array}{l}112 \\
28\end{array}$ & $\begin{array}{l}56,3 \\
53,6\end{array}$ & 1,11 & $(0,48-2,55)$ & 0,7986 \\
\hline $\begin{array}{l}\text { Inicio oportuno de la LM, antes de las } 24 \mathrm{~h} \text {. } \\
\text { de haber nacido }\end{array}$ & $\begin{array}{l}\mathrm{SI} \\
\mathrm{NO}\end{array}$ & 110 & $\begin{array}{l}54.5 \\
60,0\end{array}$ & 0,80 & $(0,35-1,81)$ & 0,5943 \\
\hline $\begin{array}{l}\text { Importancia de la LME } \\
\text { durante los primeros } 6 \text { meses de nacido }\end{array}$ & SI & $\begin{array}{l}126 \\
14\end{array}$ & $\begin{array}{l}56,3 \\
50,0\end{array}$ & 1,29 & $(0,42-3,89)$ & 0,6507 \\
\hline $\begin{array}{l}\text { Extracción de la LM para almacenar, } \\
\text { descongelar y administrar al niño }\end{array}$ & $\begin{array}{l}\mathrm{SI} \\
\mathrm{NO}\end{array}$ & 56 & $\begin{array}{l}53,4 \\
60,7\end{array}$ & 0,71 & $(0,35-1,41)$ & 0,3315 \\
\hline $\begin{array}{l}\text { Alimentación balanceada durante } \\
\text { el embarazo y la lactancia }\end{array}$ & $\begin{array}{c}\text { SI } \\
\text { NO }\end{array}$ & $\begin{array}{l}114 \\
26\end{array}$ & $\begin{array}{l}56,1 \\
54,8\end{array}$ & 1,09 & $(0,46-2,58)$ & 0,8317 \\
\hline $\begin{array}{l}\text { Beneficios de la LM y el calostro } \\
\text { para el niño }\end{array}$ & $\begin{array}{c}\text { SI } \\
\text { NO }\end{array}$ & $\begin{array}{c}107 \\
33\end{array}$ & $\begin{array}{l}57,9 \\
48,4\end{array}$ & 1,46 & $(0,67-3.20)$ & 0,3402 \\
\hline Beneficios de la LM para la madre & $\begin{array}{l}\text { SI } \\
\text { NO }\end{array}$ & $\begin{array}{l}98 \\
42\end{array}$ & $\begin{array}{l}56,1 \\
54,8\end{array}$ & 1,06 & $(0,51-2,8)$ & 0,8819 \\
\hline $\begin{array}{l}\text { Riesgo del uso de fórmulas lácteas } \\
\text { (otras leches en biberón) }\end{array}$ & $\begin{array}{c}\text { SI } \\
\text { NO }\end{array}$ & $\begin{array}{l}83 \\
57\end{array}$ & $\begin{array}{l}55,4 \\
56,1\end{array}$ & 0,97 & $(0,49-1,91)$ & 0,933 \\
\hline Otros factores antes del parto & & & & & & \\
\hline $\begin{array}{l}\text { Experiencia exitosa en dar LME a } \\
\text { otros hijos }\end{array}$ & SI & $\begin{array}{l}53 \\
87\end{array}$ & $\begin{array}{l}64,2 \\
50,6\end{array}$ & 1,75 & $(0,87-3,53)$ & 0,1183 \\
\hline Paridad & $\begin{array}{l}\text { Primípara } \\
\text { Multípara }\end{array}$ & $\begin{array}{l}72 \\
68\end{array}$ & $\begin{array}{l}54,2 \\
50,6\end{array}$ & 0,88 & $(0,45-1,71)$ & 0,7045 \\
\hline Apoyo del padre del niño & $\begin{array}{l}\mathrm{SI} \\
\mathrm{NO}\end{array}$ & $\begin{array}{l}114 \\
26\end{array}$ & $\begin{array}{l}59,6 \\
38,4\end{array}$ & 2,36 & $(0,98-5,66)$ & 0,0536 \\
\hline Embarazo planificado & $\begin{array}{c}\text { SI } \\
\text { NO }\end{array}$ & $\begin{array}{l}62 \\
78\end{array}$ & $\begin{array}{l}56,5 \\
55,1\end{array}$ & 1,06 & $(0,54-2,07)$ & 0,8756 \\
\hline
\end{tabular}

$\mathrm{n}$ : Número de casos.

LME: Lactancia Materna Exclusiva (durante seis meses).

OR: Odds Ratio, Medida de asociación (probabilidad de que una madre expuesta a un factor le de LME hasta los seis meses).

IC: Intervalo de confianza.

$\mathrm{p}$ : Significativo si es menor de 0.05 (si existe asociación). 
Tabla 3. Nivel de asociación de factores durante el parto en establecimientos de salud, Lima Centro, junio 2019.

\begin{tabular}{|c|c|c|c|c|c|c|}
\hline Factor & Condición & $\mathbf{n}$ & LME(\%) & OR & IC $95 \%$ & $\begin{array}{c}\mathbf{p} \\
\text { significancia }\end{array}$ \\
\hline \multirow{2}{*}{ Tipo de parto } & Vaginal & 77 & 63,6 & \multirow{2}{*}{1,99} & \multirow{2}{*}{$(1,01-3,94)$} & \multirow{2}{*}{0,047} \\
\hline & Abdominal & 62 & 46,7 & & & \\
\hline \multirow{2}{*}{ Contacto piel a piel madre-recién nacido } & $\mathrm{Si}$ & 116 & 58,6 & \multirow{2}{*}{1,98} & \multirow{2}{*}{$(0,81-4,84)$} & \multirow{2}{*}{0,132} \\
\hline & No & 24 & 41,6 & & & \\
\hline \multirow{2}{*}{$\begin{array}{l}\text { Madre dio de lactar a su bebé el primer día } \\
\text { de nacido }\end{array}$} & $\mathrm{Si}$ & 127 & 58,3 & \multirow{2}{*}{3,14} & \multirow{2}{*}{$(0,92-10,74)$} & \multirow{2}{*}{0,068} \\
\hline & No & 13 & 30,8 & & & \\
\hline \multirow{2}{*}{ Alojamiento conjunto madre-bebé } & $\mathrm{Si}$ & 119 & 58,0 & \multirow{2}{*}{1,84} & \multirow{2}{*}{$(0,72-4,70)$} & \multirow{2}{*}{0,202} \\
\hline & No & 21 & 42,9 & & & \\
\hline
\end{tabular}

n: Número de casos / LME: Lactancia Materna Exclusiva (durante seis meses) / OR: Odds Ratio, medida de asociación (probabilidad de que una madre expuesta a un factor le dé LME hasta los seis meses) / IC: Intervalo de confianza / p: Significativo si es menor de 0.05 (si existe asociación).

Tabla 4. Nivel de asociación de factores durante el parto en establecimientos de salud, Lima Centro, junio 2019.

\begin{tabular}{lcccccc}
\hline \multicolumn{1}{c}{ Factor } & Condición & $\mathbf{n}$ & LME(\%) & OR & IC 95\% & $\begin{array}{c}\text { P } \\
\text { significancia }\end{array}$ \\
\hline Percibe insuficiente producción & Si & 72 & 56,9 & 0,902 & $(0,46-1,76)$ & 0,763 \\
de leche & No & 68 & 54,4 & & & \\
Uso de fórmulas lácteas & Si & 58 & 24,1 & 11,175 & $(5.04-24.80)$ & $<0,001$ \\
Ambiente adecuado para dar de & No & 82 & 78,1 & & & \\
lactar & Si & 30 & 43,3 & 0,53 & $(0,23-1,20)$ & 0,126 \\
Apoyo familiar & No & 110 & 59,1 & & & 0,708 \\
Contó con visita domiciliaria del & Si & 117 & 56,4 & 1,19 & $(0,48-2,90)$ & \\
personal de salud & No & 23 & 52,2 & & & 0,415 \\
\hline
\end{tabular}

n: número de casos. / LME: Lactancia Materna Exclusiva (durante seis meses). / OR: Odds Ratio, Medida de asociación (probabilidad de que una madre expuesta a un factor le dé LME hasta los seis meses). / IC: Intervalo de confianza. / p: significativo si es menor de 0.05 (si existe asociación).

\section{DISCUSIÓN}

La prevalencia de LME encontrada en el grupo estudiado es de $46,4 \%$, esta cifra es inferior a lo reportado por ENDES $2018^{(6)}$, que señala $66,4 \%$ como promedio nacional y $61,2 \%$ para el área urbana, mientras que en el año 2016 fue de $66,7 \%$ el promedio nacional ${ }^{(14)}$. Existen reportes $s^{(1,10,11)}$ de que al inicio hay buena disposición de las madres para dar la leche natural a su hijo, pero al salir de alta de la institución donde han alumbrado, por diversas razones abandonan paulatinamente la exclusividad de la lactancia y el porcentaje de madres que llegan al sexto mes de vida de sus bebés proporcionándoles solo leche materna es muy bajo.

En nuestro estudio el $90,7 \%$ le dio de lactar durante el primer día de nacido, de ellas el 58,3\% llegó a darle hasta los seis meses la LME. En el Perú, según ENDES $2018^{(6)}, 49,7 \%$ lactó durante la primera hora de nacido, aunque hay diferencias según el área urbana $(43,4 \%)$, rural $(68,7 \%)$. Los que lactaron dentro del primer día constituyen el $92,3 \%$.

En cuanto a los factores que se presentan antes del parto, se encontró asociación estadísticamente significativa entre la asistencia a seis o más controles prenatales con la práctica de la LME hasta los seis meses. Las madres que no asisten a sus controles en algunas ocasiones se debe a que olvidan sus citas, en un estudio realizado en dos hospitales del Huaral y Chancay se encontró que el olvido de las citas estaba asociado a la adherencia de control prenatal ${ }^{(15)}$. Por otro lado ENDES 2018(6), informa que el $92,5 \%$ de gestantes, a nivel de Lima y Callao, recibieron seis o mas controles, esta situación es muy positiva ya que expresa el interés de las mujeres gestantes en cuidar su estado de salud y prever riesgos.

Aunque no se encontró significancia entre los aspectos 
tratados acerca de una lactancia exitosa durante los controles, en todos los casos, la proporción de madres que recibieron estas instrucciones fue mayor que las que no recibieron. Otros estudios como los de BellidoRoque y col. ${ }^{(16)}$ en Lima, encontraron que las madres tenían mediano conocimiento acerca de la LME. El Reglamento de Alimentación Infantil(17) establece que en cada establecimiento de salud donde se brinde atención a gestantes, debe tener conformado un Comité de Lactancia, para reforzar la información sobre los beneficios de la leche materna para el niño y para la madre a corto y largo plazo; Es importante que en todos los establecimientos de salud se cumplan estas directivas. Una publicación en Bangladesh ${ }^{(18)}$ sobre el impacto de una intervención educativa haciendo énfasis en nutrición dio muy buenos resultados para la promoción de la LME.

Durante el proceso del alumbramiento, el factor parto vaginal estuvo asociado a la LME en nuestro estudio, resultado similar encontró Silva Ocampo y col. ${ }^{(9)}$ en Chile, ya que este tipo de parto se considera normal, permite que, inmediatamente que el bebé es expulsado se le pueda acercar a la madre para un contacto precoz, estimulándolo a que busque el pezón para alimentarse por primera vez. La OMS establece que la frecuencia de cesárea(5) debe ser como máximo 15\%, sin embargo, en nuestro país se ha incrementado en los últimos años hasta 46\% para el año 2018 en el ámbito de Lima Metropolitana según ENDES 2018-Salud Materna. Esto es preocupante ya que no es recomendable la cesárea cuando no es absolutamente necesaria.

En el periodo pos parto, se encontró asociación del factor uso de fórmulas lácteas en biberones con la $\mathrm{LME}$, lo cual constituye un factor de riesgo muy alto para no dar LME. Lamberti y col. ${ }^{(20)}$ encontraron que los niños alimentados con fórmulas presentan casi quince veces más mortalidad por neumonía y enfermedades respiratorias. Rollins y col. ${ }^{(21)}$ calcularon que para el año 2019 las ventas de productos sucedáneos de la LM ascenderían a más de 70 mil millones de dólares. En general, se hace caso omiso de las recomendaciones y continúan con la promoción de sus productos en todo lugar donde puedan encontrar madres que no estén muy convencidas de los beneficios de proporcionar su propia leche a sus hijos. El Código de Comercialización de Sucedáneos de la leche ha sido actualizado recientemente ${ }^{(22)}$.

Otro estudio ${ }^{(23)}$ encontró asociación entre el abandono de la LME con el uso del biberón, sin embargo, existen mujeres que declaran que la LME es lo mejor para el bebe, pero en la práctica no lo cumplen ${ }^{(24)}$ Otro factor mencionado con frecuencia por diversos autores es la percepción de tener poca leche (hipogalactia), o que su hijo se queda con hambre como lo reportado por Ramiro-González, en España ${ }^{(25)}$, en $\mathrm{Cuba}^{(26)}$ y en Colombia ${ }^{(27)}$.

De las que manifestaron tener alguna enfermedad que le impedía dar su leche al bebé sólo fueron 8 de 140, a pesar de ello, la mitad, es decir cuatro si afirmaron haberle dado LME hasta los seis meses. Una publicación en Argentina reporta que los síntomas depresivos y de ansiedad disminuyen la probabilidad de prolongar el tiempo de lactancia hasta los seis meses ${ }^{(28)}$.

La mayoría de madres entrevistadas en nuestro estudio (78,57\%) manifestaron no tener un ambiente adecuado, es decir con poco ruido, lugar espacioso, sin interrupciones, de este grupo de madres a pesar de este inconveniente el 59,1\% dieron LME. Solo el 24,3\% de las madres manifestó haber recibido visita domiciliaria del personal de salud, de ellas el 61,8\% respondió que dio LME a su bebé por seis meses, el problema es que muchas veces por falta de personal profesional no se realizan estas visitas que constituyen la prolongación del proceso de atención a la madre. En este aspecto es importante la intervención del personal de salud que visite a la madre en su domicilio, para observar el ambiente y dar algunas recomendaciones para que se reduzcan al mínimo las situaciones de estrés.

\section{Limitaciones}

Una de las limitaciones ha sido el tamaño de muestra, considerando que uno de los tres distritos de la DIRIS Lima Centro mas afectados por la prevalencia de EDA e IRA es San Juan de Lurigancho, que es uno de los mas poblados de Lima Metropolitana.

\section{Consideraciones éticas}

El protocolo de estudio fue aprobado por el comité de ética e investigación de la Facultad de Medicina de la UNMSM, con el Acta No19-0014. Se realizó el proceso de consentimiento Informado a todas las participantes, cumpliéndose los principios fundamentales de la persona: Autonomía, Confidencialidad de sus datos, y la No maleficencia.

\section{CONCLUSIÓN}

En conclusión, se encontraron tres factores asociados a la LME: asistencia a seis controles prenatales, parto vaginal y como factor de riesgo, el uso de formulas lácteas, uno en cada espacio de tiempo, antes, durante y después del parto.

La recomendación para las instituciones de educación superior y universidades formadoras de profesionales de la salud es que en los planes de estudio existan asignaturas que fomenten y profundicen la trascendencia de la práctica de la lactancia materna 
exclusiva hasta los seis meses ya que contribuye a la mejora de la salud pública.

Por otro lado, es importante que se reduzca la proporción de partos por cesárea y que solo se realice cuando sea absolutamente necesario.

Se debe continuar también con las campañas para evitar en lo posible el uso de biberones, si bien en algunos casos puede ser necesario, no debe ser una práctica común.

\section{Correspondencia: Carmen Villarreal Verde.}

Dirección: Los Fitopatólogos 254, Lima12-Perú.

Teléfono: 959063461

Correo:cvillarrealv@unmsm.edu.pe

\section{REFERENCIAS BIBLIOGRÁFICAS}

1. Victora C, Bahl R, Barros A, Franca G, Horton S, Krasevec J, y col. La lactanci materna en el Siglo XXI: epidemiología, mecanismos y efectos a lo largo de la vida. Lancet- Primera serie Lactancia Materna 2016. p 1-16. Doi:https://doi.org/10.1016/ S0140-6736(15)01024-7

2. Cabedo R, Manresa J, Cambredó MV, Montero L, Reyes A, Gol R y col. Tipos de Lactancia Materna y factores que influyen en su abandono hasta los seis meses. Estudio LACTEM. Matronas Prof. 2019; 20(2): 54-61 https://www.federacionmatronas.org/wp-content/uploads/2019/05/54-ORIGINAL-TIPOS-LACTANCIA.pdf

3. Campiño-Valderrama SM, Duque PM. Lactancia materna: Factores e propician su abandono. Archivos de Medicina (Manizales) 2019 19(2),331-41. DOI: https://doi. org/10.30554/ archmed.19.2.3379.

4. Martínez Locio L, Hermosilla M. Razones de abandono de la Lactancia Materna en madres con hijos menores de 2 años. Mem Inst Investig Cien Salud 2017; 15(2):73-78. Doi: 10.18004/Mem.iics/1812-528/2017.015(02)73-078

5.Becerra-Bulla F, Rocha-Calderon L, Fonseca- Silva DM, Bermudez-Gordillo LA El entorno familiar y social de la madre como factor que promueve o dificulta la lactancia materna. Rev Fac Med 2015; 63(2): 217-227. DOl: http://dx.doi. org/10.15446/revfacmed.v63n2.44051

6. Instituto Nacional de Estadística e Informática (INEI).Lactancia y Nutrición de Niñas, Niños y Mujeres cap. 10 en Encuesta Demográfica y de Salud Familia (ENDES) 2018. https://www.inei.gob.pe/media/MenuRecursivo/publicaciones digitales/Est/Li b1656/index1.html

7. Perú. Ministerio de Salud. Dirección de Redes Integradas de Salud Lima Centro Oficina de Epidemiología. Sala Situacional, semana 52, año 2018. https://drive google.com/drive/folders/1h2u2igt6LXwlsd_SaO3hn7R61r4PzC_A

8. Organización Mundial de la Salud. Declaración de la OMS sobre tasa de cesárea. Programas y Proyectos. 2015. Disponible en: https://apps. who.int/iris/bitstream/handle/10665/161444/WHO_RHR_15.02_spa. pdf;jsessionid=0EFF77CE4E9E4E17809EC2F854CAE727?sequence $=1$

9. Silva P, Vargas N, León N, Duran S, Araya M, Rudman J y col. El tipo de parto ¿Podría condicionar el éxito en la lactancia materna exclusiva? Rev Esp Nut Comunitaria 2018; 24 (2): 1-8. Disponible en: http://www.renc.es/imagenes/auxiliar/ files/RENC 2018 2 2 1. S D Duran-Aguero. Tipo de_parto_y_lactancia.pdf

10. Collaborative Group on Hormonal Factors in Breast Cancer. Breast Cancer and Breastfeeding: Collaborative reanalysis of individual data from 47 epidemiologica studies in 30 countries, including 50302 women with breast cancer and 96973 women whithout the disease Lancet 2002; 360:187-95. Citado por Victora y col. DOI: https://doi.org/10.1016/S0140-6736(02)09454-0

11. Perú. Ministerio de Salud. Instituto Nacional de Enfermedades Neoplásicas. Departamento de Epidemiología y Estadística del cáncer. Registro de Cáncer de Lima Metropolitana, Incidencia y Mortalidad 2010-2012. Volumen 5. Lima 2016.http://www.inen.sld.pe/portal/documentos/pdf/banners_2014/2016/ Registro\%20de\%20C\%C3\%A1ncer\%20Lima\%20Metropolitana\%202010\%20-\%20 2012 02092016.pdf

12. Martínez-Galán P,Martín-Gallardo E, Macarro-Ruiz D, Martínez-Mar5tín E, Manrique-Tejedor J. (2016) Educación prenatal e inicio de la lactancia materna: Revisión de la literatura. Enfermería Universitaria, 14(1),54-66. http://dx.doi. org/10.1016/j.reu.2016.11.005 1665-7063/@

13. Asociación Peruana de Empresas de Investigación de Mercados (APEIM). Niveles Socioeconómicos 2018 .Lima julio 2018. http://apeim.com.pe/wp-content/ uploads/2019/11/APEIM-NSE-2018.pdf

14. Perú. Instituto Nacional de Estadística e Informática (INEI).: Indicadores de Resultados de los Programas Estratégicos. Primer Semestre 2016. https://proyectos. inei.gob.pe/endes/ppr.asp

15. Rivera Félix LM, Burgos López NH, Gómez Díaz JZ, Moquillaza Alcántara VH
Contribuciones de autoría: Los autores participaron en la génesis de la idea, diseño de proyecto, recolección e interpretación de datos, análisis de resultados y preparación del manuscrito del presente trabajo de investigación.

\section{Financiamiento: Autofinanciado.}

Conflicto de interés: Los autores declaran no tener conflicto de interés en la publicación de este artículo.

Recibido: 28 de enero 2020

Aprobado: 19 de marzo 2020
Factores asociados al acceso a la atención prenatal en los hospitales de Huaral y Chancay, Perú. Rev An Fac Med 2018;79(2):131-7. DOI: http://dx.doi.org/10.15381/ anales. v79i2.14939

16. Bellido-Roque L, Bellido Roque J. Nivel de conocimientos, creencias y actitudes sobre Lactancia Materna Exclusiva que poseen las madres puérperas en el Centro de Salud Piedra Liza, 2014. Agora Rev Cient 2016; 03(01): 300-306. DOI: http:// dx.doi.org/10.21679/arc.v3i1

17. Perú, Ministerio de Salud. MINSA. Reglamento de Alimentación Infantil Comisión Multisectorial de Promoción y Protección de la Lactancia Materna Comite Técnico Institucional para la Promoción y Protección de la Lactancia Materna en el Perú.2015. Disponible en: http://bvs.minsa.gob.pe/local/MINSA/3290.pdf

18. Nguyen PH, Kim S, Sanghvi T, Mahmud Z, Mai Tran L, Shabnam Sy col. Integrating Nutrition Interventions into an Existing Maternal, Neonatal and Child Health Program Increased Maternal Dietary Diversity, Micronutrient Intake and Exclusive Breastfeeding Practices in Blangladesh: Results of a cluster- Randomized Program Evaluation. The Journal of Nutrition 2017;2326-2337. Doi: https://doi.org/10.3945/ jn.117.257303.

19. Lamberti LM, Zakarija-Grković I, FischerWalker CL, et al. Breastfeeding forreducing the risk of pneumonia morbidityand mortality in children under two: a systematic literature review and metaanalysis. BMC Public Health 2013:13 (Supp 3:S18):S3-18. Doi: http://www.biomedcentral.com/1471-2458/13/S3/S18

20. Rollins NC, Bhandari N, Hajeebhoy $N$, et al., en nombre de The Lance Breastfeeding Series Group. Why invest, and what it will take to improve. Doi: 10.1016 / S0140-6736 (15) 01044-2.

21. Código Internacional de Comercialización de Sucedáneos de la leche Actualización 2017. Disponible en: https://apps.who.int/ iris/bitstream/handle/10665/255194/WHO-NMH-NHD-17.1-spa. pdf;jsessionid=E03233AB41 A5DB7F0C9D4F0C5A97B3B3? sequence=1

22. Camargo- Figueroa FA, La torre-La torre JF. Porras Carreño JA. Factores asociados al abandono de la lactancia materna exclusiva .Hacia la promoción de la salud. 2011; 16(1),56-72. Disponible en :http://www.scielo.org.co/pdf/hpsal/v16n1/ v16n1a04.pdf

23. Quispe-llanzo MP, Oyola-García AE, Navarro-Cancino MN, Silva Mansilla JA Características y creencias maternas asociadas al abandono de la Lactancia Materna Exclusiva. Revista Cubana de Salud Pública, 2015; 41(4),582-592. Disponible en http://scielo.sld.cu/pdf/rcsp/v41n4/spu02415.pdf

24. Ramiro González MD, Ortiz-Marrón H, Cañedo-Arguelles CA, Esparza Olcina MJ, Cortes-Rico O, Terol-Claramonte M y col. Prevalencia de la lactancia materna y factores asociados con el inicio y la duración de la lactancia materna exclusiva en la comunidad de Madrid entre los participantes del estudio ELOIN. Anales de Pediatría (Barc),2018; 89 (1), 32-43.Doi:https://doi.org/10.1016/j.anpedi.2017.09.002

25. Rivera Ledesma E, Bauta León L, Fornaris Hernández A, Flores Martínez M, Pérez Majendie R. Factores determinantes de la lactancia materna exclusiva: Policlínico "Aleyda Fernández". Revista Habanera de Ciencias Médicas [revista en Internet] 2017; 16 (6), 879-890. Disponible en: http://www.revhabanera.sld.cu/index.php/ rhab/article/view/2075

26. Mateus SJC, Cabrera AGA. (Factores asociados con la práctica de lactancia exclusiva en una cohorte de Mujeres de Cali, Colombia. Factors associated with exclusive breastfeeding practice in a cohort of women from Cali, Colombia. Colombia Médica (Cali), 2019; 50(1),50 (1),22-29. DOl:10.25100/cm.v50i1.2961.

27. Monteban MF. Factores Asociados con la Lactancia Materna en madres concurrentes a servicios de Atención Primaria en Salud en el NOA (Noroeste de Argentina). Cuadernos de la Facultad de Humanidades y Ciencias Sociales. Universidad de Jujuy, 2018;№ 54,191-212.ISSN: 0327-1471 http://revista.fhycs.unju. edu.ar/revistacuadernos. file:///D:/MIS\%20DOCUMENTOS/Downloads/298-2541 2-PB\%20(1).pdf 